awakened so as to restore the equilibrium of the slightingly, and imply both by word and conduct that higher or cortical sensory-motor reflex arc. Of course, they believe the patient to be a malingerer, and that heredity is a tremendous obstacle to overcome, but is his sufferings are entirely false and even conjured for not altogether insurmountable. Given, therefore, all the occasion. It will, therefore, be the purpose of measures to restore the physical condition. nutrition, this paper to try and correct such erroneous inference etc., of the tissues involved, the treatment of hysteria as well as place it upon its true scientific basis. resolves itself into the study and correction of the patient's sensory-motor reflexes. This involves so many obvious details that I will not occupy your time now in rehearsing them.

Reliance Building.

\section{THE RELATION OF NEURASTHENIA TO INSANITY.}

Presented to the Section on Neurology and Medical Jurisprudence at the Forty-ninth Annual Meeting of the American Medical Association, held at Denver, Colo., June 7-10, 1898.

BY JOHN PUNTON, M. D.

KANSAS CITY, MO'.

The vast increase of our knowledge of the nervous system and its application to disease has been found to be most useful in the correction of certain former erroneous inferences. Hence a complete revolution has taken place in our knowledge of its pathologic states, which has also been the means of greatly en. larging the range of its organic lesions, at the same time materially limiting the scope and significance of its so-called functional affections. In spite of our grand success, however, in unraveling the secrets of the morbid changes in both structure and function of the neuron, much still remains to be done before we can successfully cross the Rubicon that separates organic lesions from those that are of purely functional origin.

This uncertainty of our knowledge has led to the formation and maintenance of a false system of nomenclature which not only confuses but greatly hinders the progress of scientific nosography. Hence many terms have come into general use which at the time of their advent were very convenient screens to shield our ignorance, but as our knowledge increased the scope of their power and significance was greatly reduced and limited, until today a larger and fuller knowledge renders many of them futile and actual misnomers. Others again embody a generalized morbid state or condition which later investigation renders capable of scientific classification, thereby greatly limiting their former power and significance, and unless the physician is fully aware of the changes wrought by such advance, he is greatly handicapped in his interpretation of the term, and his skill in diagnosis proportionately suffers. In no branch of medicine are such mistakes more common and conspicuous than those found to be associated with lesions of the nervous system. Nor is this strange when we remem. ber its complex mechanism and the difficulty experienced in mastering the details of its scientific study in health and disease.

One of the most striking and widespread examples of mistaken identity in the true interpretation of the significance of the term is found associated with the morbid condition designated as "neurasthenia." I doubt if there is a more common and serious affection of the nervous system that is more generally misun. derstood by the profession at large than this fatigue. neurosis. It is surprising to find the gross indifference manifested on the part of many physicians toward its real nature and character. Not only do they treat neurasthenia carelessly, but they refer to it
The term "neurasthenia" was supposed to have been first used by Dr. Beard in describing a general morbid weakness or exhausted state of the nervous system. Later investigations, however, prove that his claim to priority in its use was an erroneous one. To Dr. VanDusen belongs the credit of using it as early as 1867 in his valuable paper entitled "Observation Upon a Form of Nervous Exhaustion, or Neurasthenia Culminating in Insanity," which appeared two years earlier than Beard's famous monograph. It has since been shown, however, that neither of these physicians invented the term, as it can be found in Dunglison's Medical Dictionary published in 1833. Notwithstand. ing these facts, the medical profession is under lasting obligations to Dr. Beard for the persistent manner in which he emphasized the importance of its study, thereby acquiring for himself an enduring monument in medical science. Prior to his labor in this direction, much confusion prevailed in the interpretation of a large group of symptoms which were fundamentally dependent upon a morbid weakness of the nerve centers, but which had been previously ascribed to disease of the various visceral organs involved, such as perverted conditions of the kidney, liver, stomach, uterus, etc. The result of Beard's work, however, led to a more thorough systematic study of its pathogenesis, which is still in progress, and the conclusions so far reached warrant the assertion that expresses an affection which does not limit itself to any part of the nervous apparatus, but affects it as a whole. Hence it is an extremely generalized condition, and often associated with other functional and organic diseases.

Its extremely generulized character may therefore affect the brain, spinal cord, peripheral nerves, the visceral organs, and indeed all parts of the human organism subject to nervous innervation. Consequently its symptomatology is exceedingly extensive and varied. This wide range of involvement has led to much confusion and is the responsible agent for the vague, ill-defined notions entertained by many concerning the true nature and character of neurasthenia; some physicians even going so far as to deny its existence altogether as a clinical entity. Laying aside all controversy, the consensus of opinion today by those most competent to speak declares that neurasthenia in its quintessence is a true fatigue neu. rosis characterized with an increased morbid reaction of the ganglionic nerve centers to all kinds of impressions, both mental and physical, whether slight or profound; producing an excessive nervous weakness and nervous irritability, which constitute its chief cardinal symptoms. Moreover, recent investigation demonstrates that while the various symptom groups occurring in neurasthenia are numerous and differ widely in detail, yet there are always present certain essential features that constitute a well-defined clinical syndrome. These have been termed by Charcot as neurasthenic stigmata.

All authorities agree that the cardinal symptoms of neurasthenia present well-marked evidence of weakness and irritability of the nerve centers which in themselves are expressive of fatigue. Weakness and 
irritability of the nervous mechanisms that preside over the various bodily organs are, therefore, the chief clinical phenomena, belonging to any and all varieties of neurasthenia. These may present themselves in all degrees of intensity and are usually accompanied with other symptoms which denote the particular functions of the nervous system that are involved. Hence we may have involvement of the motor, sensory, reflex, trophic, secretory, visceral and psychical mechanisms which furnish a wide and varied range of clinical phenomena. But it matters little what part of the nervous apparatus is included in the pathologic process, the two dominating factors that are present and common to all varieties, and that which defines neurasthenia as a separate and distinct clinical entity are weakness and irritability of the various nervous functions involved. They are, therefore, properly regarded as the essential primary or fundamental symptoms of the lesion while all other associated clinical evidences of involvement are termed secondary or ad. ventitious symptoms. These latter may be the out. growth of the former and are simply parts of a well. defined harmonious entity.

Neurasthenia, therefore, presents two great classes of symptoms, viz: primary and secondary or adventitious, which may affect both the physical and mental constitution in varying degrees of intensity. The skill of the physician, therefore, in its diagnosis de. pends upon his ability to differentiate the one from the other, and upon this largely hinges the prognosis. Moreover, neurasthenia is variously classified by different authors according to its etiology, symptomatology or the apparent seat of lesion. Hence we may have cerebral, spinal, traumatic, sexual, toxemic, reflex and many other forms of the disease. While no doubt this method has some advantages, yet it often proves very confusing and misleading.

A classification that recognizes neurasthenia in its entirety is to my mind, therefore, much preferable, and from this standpoint it can be reduced to two classes, viz: 1, complicated; 2 , uncomplicated neurasthenia. In the former it may be found associated with many functional as well as organic diseases, such as epilepsy, syphilis, malaria, gout, rheumatism, uterine disease, hypochondria, sexual perversion, insanity, as well as many other morbid conditions. In the latter it presents itself by a conspicious absence of any such complications, in which case it is known as simple or uncomplicated neurasthenia. The effects of either class, however, produce symptoms which may be referred to both the physical as well as mental constitution of the individual, and present a wide range of clinical phenomena that are, for the most part, subjective in character. While it is true that much speculation has been indulged in relative to its pathology, we are still compelled to admit that so far we are unable to positively declare what the actual changes of the nervous elernents are in neurasthenia.

The two important symptoms, viz: morbid irritability and morbid weakness of the nerve centers, have been shown by Hodge to be due to changes in the nerve cell itself, and that the lesion of fatigue is loss of cellular substance involving the nucleus, protoplasm and even the capsule itself. According to Dercum, this waste of nerve substance had been demonstrated by Mosso to alter the constitution of the blood, producing a twue toxemia that strongly inhibits the physiologic action of the nervous elements distributed to the voluntary muscles and other organs of the body. Let this be as it may, it is certain that the element of autotoxemia enters largely into the production of neurasthenia, and often gives rise to tissue changes that are commonly found associated with its more severe forms. The continued results, however, of nerve strain from any cause, that form so prominent a factor in American business, social, political, and professional life, operate upon the physical and mental organization in such a man. ner as to exhaust strength or vital force more rappidly than recuperation takes place. Consequently nervous exhaustion or neurasthenia ultimately ensues. More especially is this found to be true of persons inheriting a neurotic diathesis, as this renders them more susceptible to the baleful influences of the common causes of neurasthenia. Excess and abuse, of any and all kinds, with faulty methods of living, operating upon such an individual are recognized by all authorities to be potent factors in the production of neurasthenia. Dana claims that the causes of neurasthenia can be reduced to two agents, viz: bad heredity and foolish living (Twentieth Century Practice.) The reduced nervous energy that results from such causes seriously affects the visceral organs and nutritive functions of the body, which is expressed clinically by a marked weakness or inaction as well as want of vigor of all the bodily processes. A feeling of bodily illness is thus engendered that attracts the attention of the patient to himself and ultimately forms the basis of morbid fears or nosophobia. This again leads to the development of despondency, petulance, irritability, selfishness, impatience and vacil. lating conduct, all of which are marked features of the formative or incipient stage of insanity. All authorities agree that incipient insanity is attended with physical and mental changes that are indicative of nerrous exhaustion, and that clinically they are expressed by a dimunition or want of vigor of all the bodily processes. Moreover, despondency and nervous irritability are also marked featuras of early insanity. 'The relation of neurasthenia to insanity, therefore, cannot be overestimated; indeed the relation one bears to the other in a large number of cases is so very close as to almost establish a true equivalency. Kirchoff has clearly shown that certain insanities, such as melancholia, mania, paranoia and paralytic dementia may develop upon a neurasthenic basis, while Chapin, in his recent work on Insanity, just published, also declares that the larger proportion of hospital admissions received in an acute stage of insanity have a history of neurasthenia. He therefore fitly styles it the "soil out of which insanity develops." Unfortunately, too often the close relation that exists between neurasthenia and insanity is entirely overlooked and even ignored. Consequently many a curable case becomes incurable by failure on the part of the physician to recognize its true nature and character. Moreover, its close relation to hysteric states often leads the physician to treat it with contempt, under the mistaken idea that the barometric changes from day to day indicate a fraudulent basis. Nothing could be further from the truth, as there is plenty of evidence in every genuine case of neuras. thenia to explain its fluctuating tendencies even in its most simple and uncomplicated forms. One of the most important signs of neurasthenia becoming complicated with mental disorder is the manner in which the feelings, thoughts and actions of the patient ab. sorb his entire attention. This self-consciousness 
is a marked feature of many forms of neurasthenia and betrays the serious invasion of the higher mental faculties in the progress of the malady.

These concepts have been variously designated by different authors as obessions, impulsions, imperative concepts, besetments and fixed ideas. They usually consist, however, of a general feeling of anxiety, which amounts to morbid doubts and fears that later lead to impulsive acts. They irresistibly force themselves upon the individual, dominating his every thought, word and deed. In their more severe forms they constitute the various phobias, but as they present themselves in all degrees of intensity, it is the degree combined with their persistency that indicates their true seriousness. For convenience of study Regis (Sea Manual Mental Medicine, p. 262) has divided them into three classes, viz: 1 , those characterized by indecision, of which all kinds of morbid doubts are typic; 2, those characterized by all kinds of morbid fears; 3 , those characterized by morbid propensities or irresistible tendencies. Each of these is again subdivided. For instance, Ball has divided the first class, or doubters, into five subdivisions, according to the nature of their predominating ideas. Hence we have: 1. "The metaphysicians, who are especially haunted by abstract questions of all kinds, such as doubts concerning heaven, hell, the world, the deity, etc." 2. "The realists, who constantly revolve in their thoughts the lower and base details of objects, such for example as the conformation of the genital organs, copulation, etc." 3. "The scrupulous, whose doubts pertain to forms of religion, such as accusing themselves of committing the unpardonable sin, or of some theft, etc." 4. "The timorous, who are fearful of committing some indelicate action by even allowing their clothing to come in contact with that of others, etc." $\tilde{5}$. "The counters, whose doubts are manifested under the form of irresistible enumeration." Such patients never get through counting different objects, such as door knobs, the houses on the street, or their numbers, etc."

The second class, or those suffering from morbid fears or phobias, is divided into three subdivisions:1. those who have a morbid fear of all kinds of ob. jects. Its expression is extremely varied, and may involve such articles as glass, knives, pins, guns, thunder, flowers, besides certain kinds of food and drink. 2. Those who have a morbid fear of places, elements or diseases. Such patients are morbidly fearful of high places, bridges, streets, churches, theaters, rivers, beside a dread of all kinds of diseases, such as heart disease, liver disease, cancer, etc. 3. Those who have a morbid or irresistible propensity to steal, lie, cheat, drink, blaspheme, set on fire, or abuse the sexual organs. All of these various states are ably shown by Regis to be due to lesions of the will and are often associated with neurasthenia.

In a limited degree they are commonly present in health; indeed very few of us escape the presence of doubts, fears or impulses as isolated sudden thoughts, but which are controlled by the dominating power of inhibition. For instance, it is a common experience for persons looking from a height to be tempted to jump down; or in walking on the street are suddenly siezed with a desire to count the houses or numbers on the doors, etc. But these sudden impulses are easily resisted or controlled, but when they appear as a complication of neurasthenia, they are often morbidly persistent and get beyond the control of the will. In their exaggerated forms, they therefore constitute a serious symptom and may become the dominant feature of a grave form of insanity. In all cases, however, where their presence can be demonstrated, they are to be regarded as marks of true degeneracy, often remaining latent for years, to be brought to the surface by some sudden or severe shock to the nervous system; such as trauma, or severe mental and physical strains, beside excesses of all kinds. That such states also often accompany insanity is generally recognized, but $I$ repeat it is their intensity combined with their persistency that indicate their true serionsness. Moreover, there can be little doubt that certain crimes are due to such sudden impulses which arise in the minds of persons who are not ordinarily regarded as insane, and which the subjects are wholly unable to resist. Hence they are truly irresponsible and should be given the benefit of the doubt. From the fact that such symptoms are so frequently seen in both neurasthenia and insanity, the close relation that exists between the two cannot be overestimated. Indeed, all the evidence proves conclusively that neurasthenia in its more profound types presents many symptoms in common with true insanity, and the line of demarkation that separates the one from the other is at times as dimly drawn as that which exists between sanity and insanity, physiology and pathology.

Moreover, the teaching of our text-books in regard to their differential diagnosis is often very misleading, for we are taught to believe that in neurasthenia the morbid fears can be removed by a process of reasoning, and that the patient can be convinced of his mistaken ideas; while in true insenity no amount of reasoning succeeds in convincing the patient of his mistaken notions. If this was taken as a criterion, many a neurasthenic patient would be regarded as suffering from insanity, when as a matter of fact his neurasthenic condition had not reached that extreme degree of mental impairment that is synonymous with true insane delusions although the morbid fears apparently resist all forms of reasoning. It must be confessed, however, that when the morbid doubts or fears reach a certain degree of intensity, combined with persistency, they then often become the equivalent of true insane delusions and should be treated accordingly.

That many cases of neurasthenia become thus complicated is clear to all, and the importance of the close relation that exists between neurasthenia and insan. ity, I repeat again, cannot be overestimated; indeed it often requires at best much practical knowledge and experience to recognize the appearance of one and the disappearance of the other.

In conclusion I wish to add that in the preparation of this paper I desire to acknowledge my indebted. ness to such authors as Regis, Dercum, Dana, Gray, Kirchoff, Clouston, Blandford, Diller and many others, beside cullings from recent neurologic and psychologic journalistic literature.

DIscussion.

Dr. Meyer-The daily work in the hospitals for the insane leads one continually to the following dilemma : that we either have to give up making diagnoses and take everything as diffused manifestations of sickness or disease, in which case we would be inclined to let neurasthenia be just one of the initial symptoms of insanity-something that may or may not go over into insanity; or, we can take the plan which I am more inclined to follow for clinical reasons, viz., endeavor to 
single out those forms of neurasthenia which we know are initial stages of certain forms of disease, and try to keep those separate and apart from neurasthenia that always remains neurasthenia without ever going over into insanity. Dr. Maclean has made a classification of insanity in which he takes neurasthenia to be the initial symptom of all general insanity, even of general paralysis. If we do that we give up all clinical method. The fact that symptoms in the beginning of general paralysis may be those of neurasthenia. should not induce us to say that that is the sameneurasthenia which may exist independently and never go over into general paralysis except after previous syphilitic infection. For clinical reasons, therefore, in working out problems, I should hesitate very much to allow neurasthenia to take quite as broad a field as Dr. Punton gave it. We have to distinguish besides, the simple neurasthenic constitution: then the real fatigue neurosis; then the initial stages of definite forms of disease, such as general paralysis, and such as periodic insanities or any special form of insanity. I admit that it is at present almost impossible to say at the start in certain forms of neurasthenia what it is. I think that it is easy enough to distinguish the constitutional neurasthenia from an acute fatigue neurosis; it is also rather easy to distinguish neuras thenia that is the beginning of general paralysis; but, nevertheless, there will be a great number of cases that will lead us into the habit of looking upon neurasthenia as that dif fused field into which we are inclined to throw anything that we can not classify any where else.

Dr. H. S. Drayton of New York-My first acquaintance with this Protean form of neurosis was derived from reading certain pamphlets by Dr. Beard of New York. I think it is to Dr. Beard that we owe the term "neuras thenia." Some eighteen or twenty years ago he published a volume entitled "A merican Nervousness," in which he described a train of symptoms that seemed peculiar to American civilization, and a little later he came out with this term "neurasthenia." He described the peculiar category of symptoms which were included under this form as having a particular relation to our American climate and civilization, and since have heard a goud deal about the Denver climate of late I have been wondering whether or not there is anything here that is at all curative of neurasthenia. Now it seems to me that in the effort to make a differential statement so that we can determine at all what there is peculiarly its own in neu rasthenia, we might ascribe to that form of nerve disease a capricious category of symptoms ; that is, that neurasthenia is indicated more by caprices of habit, caprices of mental expression. The causative factors are to be looked at from the point of view of the-individual's heredity and life, as in all forms of nerve disease. Not long ago. Dr. Spitzger, whose name I think is very well known in this circle, delivered a very elaborate opinion with regard to the difference to be drawn between neurasthenia and melancholia, and in a nut shell, he seemed to be of the opinion that most neurasthenic troubles were traceable to specific cause. Looking at the sub. ject from one aspect, that is, the number of cases of young people affected, young men especially affected by neurasthenia it strikes me from my own observation that the great majority of these causes are found among the comparatively young; so that it may be termed a trouble or affection of those chiefly under 40 years of age. Now, can we fairly discriminate; I might ask, can we draw a very fine line of demarkation be. tween neurasthenia and melancholia? It strikes me that we cannot. From what I have heard from the late reader in that most admirable paper, my opinion is confirmed in regard to the relation of neurasthenia to insanity. Accepting that view, we see the necessary relation to melancholic patients.

Dr. TomLinson-The more experience I have in the observa tion of cases of ineanity the more am I confirmed in the conclusion that it is about time to call a halt in multiform clasei fications and diagnoses, and while we can say that the average case of insanity presents certain physical conditions which have for their outward manifestations the characteristics of neurasthenia, still, at the same time, this is present in all cases of insanity, and there is no difference between the neurasthenic manifestations of an insane person and the neurasthenic mani festations of a person who is not insane. So far as trying to make a relation between them is concerned, it only ends in confusion. While the insane person may in the earlier stages, or may, all through the outbreak of mental disturbance, manifest neurasthenic symptoms, still the neurasthenic symptoms have nothing to do with the insanity, nor does the insanity have anything to do with the neurasthenia. It is a matter of relation rather than of causation. It is not those cases in which neurasthenia is must marked that afterward become insane. The same holds true with regard to the other conditions which are supposed to differentiate forms of insanity, and so far as their practical care and treatment are concerned, in the effort to make a diagnosis and $t$ "s put this case or that case into a marked classification, this has a tendency to obscure and interfere with our treatment. Furthermore, there is not, so far as I know, any pathologic difference. and we have to go further back in the history of the case to determine just what the significance of these manifestations is. It seems to me it lies in the difference of degree of instability or defect in the individual who becomes insane or neurasthenic, and that we can get a clearer and more definite idea of the subject by recognizing the neurasthenic as representing a certain degree of instability. So long as we do not bear this in mind, the more we try to classify, and the more we try to understand these cases, the more will we become confused. In my own work I have practically given up attempting to make a diagnosis or attempting to classify one case from another, because I see that one case, although it may manifest a certain number of symptoms which might place that individual case in a certain classification, still during the period of the illness or mental disturbance there will be a whole host of other symptoms manifest themselves which would seem to put the case in another category. So, while it may for a time be of interest to classify them, so far as the patient is concerned, it is important to recognize that it does not make any difference what their condition is - what we have to deal with is the insanity, and back of that is the degree of the defect of the individual, and upon the degree of that defect will depend the diagnosis.

DR. HOPPE - I believe that the view which the individual members buld depends upon the standpoint from which they see their cases. whether in insane asylums or in general practice. Personally I have never seen but one case of neurasthenia pass into insanity. I believe that these cases are very sharply divided and identified, and 1 do not think that the pure form of neurasthenia will ever pass into insanity. For instance, take that class of neurasthenics who have fixed ideas. Their judgment may be wrong in this respect, but in all other respects it is right. It is not so much an error of judgment as it is hased upon a fear which can be classed among the photophohias or neurasthenias which necur in those cases of neurasthenia which are conetitutional in character and based upon hereditary defects. I believe there is a very sharp line of demarkation between neurasthenia and melancholia. It is true that in neurasthenia we have depressed states; but that in my estimation does not constitute melancholia. I helieve that the fundamential idea of melancholia is the idea of selfaccusation: that the patient takes some point in his past history, real or imaginary, which he accuses himself of having committed, and which he looks upon as the cause of all his mental pain and suffering, whereas we never see that in the depressed form of neurasthenia.

Dr. Eskridie- I shall have to differ from my friend Dr. Hoppe. I have seen quite a number of cases of neurasthenia pass in to melancholia. During the past fourteen years I have been able to follow my cases from the clinic room and the office to the insane asylum, having the privilege of visiting patients there from time to time during that period. I have thus been able to follow a great many cases of neurasthenia. There is a very close relation between neurasthenia and insanity. I doubt very much whether any one can positively say that a given case of neurasthenia will not pass into melancholia. I doubt very much whether anyone cay say with any degree of certainty that this particular case of neurasthenia is not melancholia. A great many cases of melancholia begin with neurasthenic symptoms. Whether that passes into insanity depends upon several causes, but two of the principal are the hereditary condition of the patient that is the nervous stamina and the man's hands into which the patient falls. There is certainly a very strong personal element in treating these cases of neurasthenia and beginning cases of melancholia. That person who can inspire confidence; that person who can make his patient feel that he or she is going to get better, whether he is a good physician or not, will have more effect than a second physician who has not personal magnetism. I do not believe that we ought to try to draw a hard and fast line between neurasthenia and melancholia.

Dr. John Punton, Kansas City-I bave nothing more to say except that $I$ appreciate highly the kind manner in which my paper has been received. and from the remarks that have been made it is evident that we are not altogether settled in our opinions as to whether there is a relation between neurasthenia and insanity or not. As far as I am concerned, my position is the same as Dr. Eskridge's. 